\title{
Effect of damage-related microstructural parameters on plate tearing at steady state
}

\author{
Tekoglu, C.; Nielsen, K. L.
}

Published in:

European Journal of Mechanics, A/Solids

Link to article, DOI:

10.1016/j.euromechsol.2019.103818

Publication date:

2019

Document Version

Peer reviewed version

Link back to DTU Orbit

Citation (APA):

Tekoglu, C., \& Nielsen, K. L. (2019). Effect of damage-related microstructural parameters on plate tearing at steady state. European Journal of Mechanics, A/Solids, 77, [103818].

https://doi.org/10.1016/j.euromechsol.2019.103818

\section{General rights}

Copyright and moral rights for the publications made accessible in the public portal are retained by the authors and/or other copyright owners and it is a condition of accessing publications that users recognise and abide by the legal requirements associated with these rights.

- Users may download and print one copy of any publication from the public portal for the purpose of private study or research.

- You may not further distribute the material or use it for any profit-making activity or commercial gain

- You may freely distribute the URL identifying the publication in the public portal

If you believe that this document breaches copyright please contact us providing details, and we will remove access to the work immediately and investigate your claim 


\title{
Effect of Damage-Related Microstructural Parameters on Plate Tearing at Steady State
}

\author{
C. Tekog̃lua, ${ }^{\mathrm{a}, *}$, K.L. Nielsen ${ }^{\mathrm{b}}$ \\ ${ }^{a}$ Department of Mechanical Engineering, TOBB University of Economics and Technology, Sögütözü, \\ Ankara, 06560, Turkey \\ ${ }^{b}$ Department of Mechanical Engineering, Solid Mechanics, Technical University of Denmark, \\ DK-2800 Kgs. Lyngby, Denmark
}

\section{Abstract}

The tearing of ductile metal plates can take place in three distinctly different modes: cup-cup, cup-cone, and crack slanting, but they are often observed in combination. It is well established that all tearing modes are governed by nucleation, growth, and coalescence of voids at the micron scale. What controls the shift between different tearing modes is, however, unclear and a micro-mechanics-based investigation is launched here to shed light on the issue. The present work takes as a starting point the hypothesis that the volume fraction, (average) size, and distribution of second phase particles, which act as void nucleation sites, are the key microstructural parameters that determine the tearing mode. In accordance with this hypothesis, the plates are modeled here by embedding randomly distributed void nucleation sites in a homogeneous matrix material. A parameter study is performed, and by adjusting the number, size and distribution of the nucleation sites in the fracture process zone, a shift in the tearing mode is achieved: a low number of small (relative to the plate thickness) randomly distributed particles link up in a void-by-void-type failure, whereas bigger particles, or a large number of small particles, can facilitate multiple void interactions. The present work also demonstrates that, for plates with intermediate or low volume fraction of nucleation sites, the localization of deformation in a macroscopic band precedes the microscopic localization that eventually links the voids. However, the two modes of plastic flow localization occur simultaneously for large volume fractions.

Keywords: Ductile failure, Gurson model, Shear band, Void coalescence, Finite element method

*Corresponding author. Tel.:+90 3122924229 ; fax:+90 3122924091 .

URL: cihantekoglu@etu.edu.tr; c.tekoglu@gmail.com (C. Tekog̃lu) 


\section{Introduction}

Intermetallic inclusions are often deemed responsible for the nucleation of the microvoids that, upon loading, grow to coalesce and eventually form the micro-cracks leading to material separation in ductile plate tearing. Distinct crack surface morphologies such as slanting, cup-cup, and cup-cone are observed for extensive crack growth in metal plates; see Fig. 1. The nucleation, growth, and coalescence of voids have been studied in great detail, and with the increasing computational resources, the chain of events are now being linked in ever more complex numerical analyses (see e.g. the reviews by Tvergaard, 1990; Benzerga and Leblond, 2010, and the references therein). An understanding of the interplay between different mechanisms and length scales is, however, still in a maturing stage and key details remain to be unraveled and exploited for the creation of new and better materials. It is reported in the literature that plates fabricated from high-strength age-hardened aluminum alloys (Irwin et al., 1958; Knott, 1973; Li and Siegmund, 2002), and high-strength steels (Broek, 1986) exhibit slanted crack growth, while plates fabricated from low-strength metals such as stainless steel, mild steel, 6082-O and NS4 aluminum alloys, brass, bronze, lead, and zinc systematically show a cup-cup fracture profile (Pardoen et al., 2004), for several different plate thicknesses. Despite this insight, the transition between the tearing modes remains to be fully understood, and nowhere is a conclusive answer found to the question: why does a propagating crack choose one tearing mode over the others?

The current approach to ductile plate tearing in the literature, based on Gurson-like porous plasticity finite element (FE) models with a homogeneous material distribution in the entire fracture process zone (FPZ), cannot provide an unambiguous answer to the above question. For a sufficiently fine discretized plate with a homogenized porosity distribution, the crack propagates in a $45^{\circ}$ orientated shear band for a wide range of material properties (Mathur et al., 1996; Felter and Nielsen, 2017; Andersen et al., 2018a). In fact, lowering the initial porosity and/or increasing the hardening capacity of the plate material while keeping the mesh unchanged can lead to a shift from slant to flat crack propagation. However, this shift is merely an artifact of the discretization; often one can make the crack slant again upon further mesh refinement. Besson et al. (2003) presented a detailed 2D study on the effect of discretization, and demonstrated 
that the mesh type has a great influence on the Gurson model's ability to describe slant crack propagation (see also Nielsen and Hutchinson, 2012).

It is well established that void nucleation becomes more and more difficult with decreasing second phase particle size (e.g Gurland, 1972; Lewandowski et al., 1989; Dighe et al., 2002), and that the presence of different families of particles with different sizes and/or void nucleation resistances have profound effects on void nucleation (e.g Marino et al., 1985; Bron et al., 2004; Asserin-Lebert et al., 2005). In the present work, the non-homogeneous distribution of the damage related microstructure is accounted for in terms of the spatial distribution, number and size of second phase particles, i.e. the void nucleation sites. The hypothesis is that a large number of closely packed nucleation sites will facilitate multiple void interaction, whereas fewer nucleation sites will link up in a void-by-void fashion. Therefore, changing the spatial distribution, number and/or size of the void nucleation sites in a metal plate, while keeping all other mechanical properties and loading conditions unchanged, is expected to lead to a change in the fracture surface morphology. Related to this is the discussion on the sequence of localized deformations at different length scales (also introduced in Tekog̃lu et al., 2015). One important finding of the present paper is that, macroscopic band formation does not necessarily occur simultaneously with, but it can precede, microscopic localization at stress triaxialities below 1, unlike the conclusions arrived at in Tekog̃lu et al. (2015).

The paper is structured as follows. The problem formulation and modeling assumptions are laid out in Section 2, and the numerical methods are presented in Section 3. Results are given in Section 4, which is followed by a discussion and concluding remarks in Section 5 .

\section{Problem formulation}

The schematic in Fig. 2a shows a ductile plate that tears under mode I loading. The dimensions of the plate in the $x_{2} x_{3}$-plane are much larger than its thickness in the $x_{1}$-direction, $W$. All the cross sections perpendicular to the crack growth direction (the $x_{3}$-direction in Fig. 2a) experience the same deformation history at steady state, such that: (1) a local thinning first develops far ahead of the crack tip, while the regions out- 
side the thinning zone unload elastically, (2) the local thinning intensifies and damage develops, (3) plastic deformation further localizes in deformation bands contained in the local thinning region, and (4) a macro-crack propagates in the deformation bands through coalescing voids, which finally leads to fracture of the cross section. The mode I tearing problem considered here is similar to that investigated in Nielsen and Hutchinson (2012); Andersen et al. (2018b), where large-scale 3D steady-state plate tearing is boiled down to an approximate 2D plane strain setup. The elastic unloading domain above and below the thinning region essentially restricts the deformation in the FPZ such that a plane strain condition can be assumed near the crack tip (an assumption supported in a recent full 3D study by Andersen et al., 2018a). Thus, in the 2D plane strain setup, a cross section in the $x_{1} x_{2}$-plane with an initial aspect ratio of $H_{0} / W_{0}$ is imagined to be cut from the plate, and loaded in tension along the $x_{2}$-direction to mimic a far-field mode I loading (see Fig. 2b). The difference in the present work is that, rather than restricting the analysis to a homogeneous porous material as in Nielsen and Hutchinson (2012); Morgeneyer et al. (2014); Andersen et al. (2018b), a population of "discrete void nucleation sites" which represents the second phase particles in a real material, are embedded into the FPZ that evolves in the region specified by its initial height, $h_{0}$, in Fig. $2 \mathrm{~b}$. The idea of modeling second phase particles as void nucleation sites has been successfully implemented in the previous literature, see e.g. Srivastava et al. (2014); Osovski et al. (2015); Morgeneyer et al. (2016); Srivastava et al. (2017). The elastic unloading domain above and below the FPZ (the light gray regions in Fig. $2 \mathrm{~b}$ ) is assumed to be non-porous and deforms by $J_{2}$-flow plasticity. In this way, the adopted setup allows microstructural parameters such as the spatial distribution, number, and relative size (with respect to the plate thickness) of the second phase particles to enter the analysis. In Fig. 2b, for example, the number of randomly distributed void nucleation sites, $N_{\mathrm{p}}$, is equal to 50 and all nucleation sites have the same radius, $R_{\mathrm{p}}$. A background porosity, which has been introduced to allow neighboring sites to eventually coalesce, is allowed to nucleate between nucleation sites. In this way, the background porosity represents a secondary population of particles with a much smaller average size than the primary population located in the nucleation sites, which is typical for metallic alloys. The Gurson-Tvergaard - Needleman (GTN) 
porous plasticity model is employed to predict the porosity evolving from the void nucleation sites as well as the macroscopic material separation that develops across the plate thickness. The FE model corresponding to this $2 \mathrm{D}$ framework enables a comprehensive parameter study of the changes in the fracture surface morphology when the damage related microstructural parameters are varied, see Section 3.

\section{Model: constitutive relations and finite element formulation}

The FE calculations in this paper are performed by using the commercial software ABAQUS/Explicit, version 2016. In this section, the constitutive relations and the FE formulation are only briefly introduced and the reader is referred to ABAQUS (2016) for further details.

\subsection{Constitutive relations}

Nucleation, growth, and coalescence of voids in the FPZ are accounted for by the GTN porous plasticity model (see e.g. Tvergaard, 1981), which is based on the pioneering work of Gurson (1977). In the notation of ABAQUS, the yield surface for the GTN model is given by:

$$
\Phi=\left(\frac{q}{\sigma_{\mathrm{y}}}\right)^{2}+2 q_{1} f^{*} \cosh \left(-q_{2} \frac{3 p}{2 \sigma_{\mathrm{y}}}\right)-\left(1+q_{3}\left(f^{*}\right)^{2}\right)=0 .
$$

Here, $q$ is the effective von Mises stress for the Cauchy stresses; $\sigma_{\mathrm{y}}\left[\bar{\varepsilon}_{\mathrm{m}}^{\mathrm{pl}}\right]$ is the yield stress of the fully dense matrix material as a function of the equivalent plastic strain in the matrix, $\bar{\varepsilon}_{\mathrm{m}}^{\mathrm{pl}}$; the hydrostatic pressure is $p$; and $q_{1}, q_{2}$, and $q_{3}$ are fitting parameters introduced by Tvergaard (1981). The three fitting parameters, respectively, account for the interaction between voids, void shape changes, and the effect of stress triaxiality on void growth during loading. Finally, $f^{*}[f]$ is a function of the void volume fraction, $f$, and is given by:

$$
f^{*}= \begin{cases}f & \text { if } f \leq f_{\mathrm{c}}, \\ f_{\mathrm{c}}+\frac{\bar{f}_{\mathrm{F}}-f_{\mathrm{c}}}{f_{\mathrm{F}}-f_{\mathrm{c}}}\left(f-f_{\mathrm{c}}\right) & \text { if } f_{\mathrm{c}}<f<f_{\mathrm{F}}, \\ \bar{f}_{\mathrm{F}}=\frac{q_{1}+\sqrt{q_{1}^{2}-q_{3}}}{q_{3}} & \text { if } f \geq f_{\mathrm{F}},\end{cases}
$$


where $f_{\mathrm{c}}$ and $f_{\mathrm{F}}$ are the two values of the void volume fraction that correspond to the onset of void coalescence and to the total loss of stress carrying capacity at a material point, respectively. Once all the material points in an element fail (with $f \geq f_{\mathrm{F}}$ ), the element is automatically removed from the mesh by ABAQUS/Explicit (see Section 3.2 for details on the element formulation).

In the GTN model, the total change in the porosity, $\dot{f}$, equals the sum of the change due to newly nucleating voids, $\dot{f}_{\text {nucl }}$, and that due to growth of the existing voids, $\dot{f}_{\mathrm{gr}}$, such that: $\dot{f}=\dot{f}_{\text {nucl }}+\dot{f}_{\text {gr }}$. In ABAQUS/Explicit, void nucleation is strain controlled, $\dot{f}_{\text {nucl }}=A\left[\bar{\varepsilon}_{m}^{p l}\right] \dot{\varepsilon}_{m}^{p l}$, and the probability density function, $A\left[\bar{\varepsilon}_{\mathrm{m}}^{\mathrm{pl}}\right]$, represents a Gaussian bell distribution for the nucleation strain:

$$
A\left[\bar{\varepsilon}_{\mathrm{m}}^{\mathrm{pl}}\right]=\frac{f_{\mathrm{N}}}{s_{\mathrm{N}} \sqrt{2 \pi}} \exp \left[\frac{-1}{2}\left(\frac{\bar{\varepsilon}_{\mathrm{m}}^{\mathrm{pl}}-\varepsilon_{\mathrm{N}}}{s_{\mathrm{N}}}\right)^{2}\right],
$$

where $\varepsilon_{\mathrm{N}}$ and $s_{\mathrm{N}}$ are, respectively, the mean value and the standard deviation for the distribution, and $f_{\mathrm{N}}$ is the total volume fraction of the nucleated voids. The assumption of a Gaussian bell distribution for void nucleation indirectly accounts for the fact that the critical strain for void nucleation is a function of the size, shape, and orientation of the second phase particles giving birth to voids. The growth of existing voids occurs through the (incompressible) plastic deformation of the surrounding matrix material, and the void growth equation directly follows from the conservation of mass in the matrix: $\dot{f}_{\mathrm{gr}}=(1-f) \dot{\varepsilon}_{\mathrm{kk}}^{\mathrm{pl}}$. For $f^{*}=f=0$, the material is fully dense, and the GTN yield surface is identical to the von Mises yield surface.

In the present study, it is assumed that the undamaged material, both in the matrix and at the nucleation sites, follows a true stress-logarithmic strain power hardening relationship described as:

$$
\sigma= \begin{cases}E \varepsilon & \text { if } \varepsilon<\varepsilon_{0} \\ \sigma_{0}\left(\frac{\varepsilon}{\varepsilon_{0}}\right)^{N} & \text { if } \varepsilon \geq \varepsilon_{0}\end{cases}
$$

where $\sigma_{0}$ is the initial yield stress, $E$ is Young's modulus, $N$ is the hardening exponent, and $\varepsilon_{0}=\sigma_{0} / E$. 


\subsection{Finite element (FE) formulation}

The FE calculations are conducted using the commercial software ABAQUS/Explicit, in a finite strain setting. Fig. 3 shows a typical FE mesh for the plate tearing problem described in Section 2. Before applying the load, the aspect ratio of the plate section is $H_{0} / W_{0}=4$ for all the calculations, where $H_{0}$ is the initial height of the plate section and $W_{0}$ the initial plate thickness. The domain specified by the initial height, $h_{0}=H_{0} / 2$, where the FPZ will evolve, is finely discretized by using square elements having a normalized edge length of $L_{\mathrm{e}} / W_{0}=10^{-3}$. The mesh size gradually increases outside the FPZ, toward the top and bottom boundaries, where an unstructured mesh containing both quadrilateral and triangular elements is accepted. In the domain of the FPZ, there is a total of $N_{\mathrm{p}}$ void nucleation sites, two of which are visible in the magnified view given in Fig. 3. The void nucleation sites have a circular shape with radius $R_{\mathrm{p}}=k L_{\mathrm{e}}$, where $k$ is an integer assumed to be the same for all the nucleation sites in a plate. It is assumed that the individual void nucleation sites are non-overlapping and do not intersect the cross-section boundaries. The adopted fine mesh allows a large number of non-overlapping void nucleation sites in this zone: for $k \leq 9, N_{\mathrm{p}}=100$ non-overlapping nucleation sites can easily be randomly distributed. The quadrilateral elements in the mesh, including those in the FPZ, are of type CPE4R (four-noded, bilinear, reduced integration with hourglass control), while the triangular elements are of type CPE3 (three-noded, linear); see ABAQUS (2016) for detailed element properties.

The plate is assumed to be non-porous in the undeformed configuration, with uniform mechanical properties throughout, representing a typical aluminum alloy: Young's modulus, $E=70 \mathrm{GPa}$; Poisson ratio, $\nu=0.3$; density, $\rho=2700 \mathrm{~kg} / \mathrm{m}^{3}$; initial yield stress, $\sigma_{0}=300 \mathrm{MPa}$; and a power-law hardening exponent, $N=0.1$. The elastic unloading regions above and below the FPZ are taken to remain non-porous during the entire loading history, whereas two different sets of porous plasticity parameters are required inside the FPZ: one for the void nucleation sites (superscript "ns") and one for the matrix (superscript "m") surrounding them. In this study, all the parameters in both sets are taken to be the same $\left(\varepsilon_{\mathrm{N}}^{\mathrm{ns}}=\varepsilon_{\mathrm{N}}^{\mathrm{m}}=10^{-2}, s_{\mathrm{N}}^{\mathrm{ns}}=s_{\mathrm{N}}^{\mathrm{m}}=(1 / 3) \times 10^{-2}\right.$, $\left.f_{\mathrm{c}}^{\mathrm{ns}}=f_{\mathrm{c}}^{\mathrm{m}}=10^{-2}, f_{\mathrm{F}}^{\mathrm{ns}}=f_{\mathrm{F}}^{\mathrm{m}}=5 \times 10^{-2}\right)$ except for the total volume fraction of the nucleated voids, $f_{\mathrm{N}}^{\mathrm{ns}}=8 \times 10^{-3}, f_{\mathrm{N}}^{m}=10^{-5}$. 
In the present work, the intention is not to enter the regime where the inertia effect will become important. Even so, the explicit (but not the implicit) solver of ABAQUS is used for two reasons: i) it automatically removes failed elements and allows the simulation to represent the separation of the plate cross section, and ii) the dynamics helps to stabilize element failure, and by exploiting mass lumping (HRZ), the calculations are easily parallelized. The price to pay for these advantages is that great care must be taken to perform the analyses at sufficiently low deformation rates that the model response can be considered quasi-static. It is, here, worth mentioning that even for very low deformation rates, such convergence has been hard to achieve for some of the microstructures considered; for convergence, the kinetic energy in the system has to be much lower than the traditional $10 \%$ of the total energy in the system. In fact, the material parameters as well as the mesh size introduced above are chosen in a such way that they allow distinguishing between different crack surface morphologies, while the total CPU time of one FE calculation for a single plate is less than 24 hours. The FE calculations are performed on an HP Z420 workstation, running four central processing units in parallel. The effect of material parameters on the results is further discussed in Section 5.

\section{Results}

The main goal of this study is to investigate the effects of the spatial distribution, number, $N_{\mathrm{p}}$, and the relative size, $R_{\mathrm{p}}=k L_{\mathrm{e}}$, of the second phase particles (acting as void nucleation sites) in relation to the fracture surface morphologies observed in plate tearing. The intervals for these key parameters, considered in the FE calculations, are: $N_{\mathrm{p}} \in\{10,25,50,75,100\}$ and $k \in\{3,6,9,15,18\}$, while all other material and geometrical parameters remain fixed. All possible $N_{\mathrm{p}}-k$ combinations are analyzed, and three realizations with different spatial distributions of nucleation sites are considered for each combination (in all $75 \mathrm{FE}$ calculations are conducted). The results are presented below, by starting first with the detailed results for the two extreme cases with $N_{\mathrm{p}}=10, k=3$ (Figs. $4-5$ ) and $N_{\mathrm{p}}=100, k=18$ (Figs. 6-7), for which the fracture surface morphologies are distinctly apart. Attention is hereafter focused on the transition between the different fracture surface morphologies (Figs. 8-11). 


\footnotetext{
${ }^{1}$ Throughout the results, attention is focused on the developed void volume fraction as this is a clear indicator of plastic flow localization in the current model setup, see Appendix.
} 
material parameters are kept constant and $N_{\mathrm{p}}=10, k=3$. The deformation and the complex sequence of events leading to fracture for P2 and P3 are the same as for P1; thinning is visible for all three plates, and they all have a cup-cup-like fracture surface morphology. The main difference in the fracture process of the three plates appear to be that, unlike in $\mathrm{P} 1$, the crack developed through more than one nucleation site in P2 and P3. However, the stress-strain curves for the three plates largely overlap until the onset of localized deformation (indicated by an asterisk $(*)$ in Fig. 5c), and even the onset of localized deformation matches up for P1 and P3. In contrast, P2 falls short of reaching the overall strain predicted for P1 and P3, but this is tied to the less pronounced development of the necking region.

Detailed results for the extreme case of a large number of big void nucleation sites $\left(N_{\mathrm{p}}=100\right.$ and $\left.k=18\right)$ are presented in Fig. 6 . As in any other case analyzed, the void nucleation starts at the onset of plastic deformation. However, in contrast to the case of a few small void nucleation sites $\left(N_{\mathrm{p}}=10, k=3\right)$, the deformation quickly localizes in a macroscopic shear band at the onset of thinning, connecting multiple nucleation sites (see Fig. 6a). The tearing crack thereby propagates through the failure of the elements located in the most energetically favorable shear bands, and this leads to an overall slanted fracture surface morphology (see Fig. 6b). It is apparent from Fig. 7 that changing the realization of randomly distributed void nucleation sites while keeping all other material properties fixed (including $N_{\mathrm{p}}=100, k=18$ ) does not affect the overall fracture surface morphology, although it shifts the location of the crack. Moreover, the stress-strain response is represented by (nearly) overlapping curves. In fact, the curves largely coincides with the response of P1 (with $N_{\mathrm{p}}=10, k=3$ ) until the onset of localized deformation (see Fig. 7d).

Figure 8 shows the developed void volume fraction in the FPZ at the final step of fracture for four different plates all with small void nucleation sites $(k=3)$, while the total numbers in each plate are $N_{\mathrm{p}}=25$ (in Fig. 8a), $N_{\mathrm{p}}=50$ (in Fig. 8b), $N_{\mathrm{p}}=75$ (in Fig. 8c), and $N_{\mathrm{p}}=100$ (in Fig. 8d), respectively. Considering the plates with $N_{\mathrm{p}}=10$ given in Fig. 5 as well, a gradual shift in the fracture surface morphology, first from cup-cup (Figs. 4, 5, and 8a) toward cup-cone (Figs. 8b and 8c), and later toward slanted (Fig. 8d) can be observed when increasing the number of randomly distributed void 
nucleation sites. Companion results for four different plates with big void nucleation sites $(k=18)$ are shown in Fig. 9. Only the location of the crack is here shifted by changing the random distribution, whereas all the plates display a slanted fracture surface morphology. In fact, among 15 plates with $k=18$ and $N_{\mathrm{p}} \in\{10,25,50,75,100\}$ analyzed in this study, including those for which the results are not shown here, the crack is predicted to be clearly slanted for 12 , clearly cup-cone for 1 , and a mixture of the three morphologies for the remaining 2 . It is also noteworthy that the amount of diffuse thinning as well as the value of the macroscopic fracture strain decrease with increasing $N_{\mathrm{p}}$, for both $k=3$ and $k=18$.

Additional results are presented in Figs. 10 and 11 to further clarify the effect of the size of the void nucleation sites on the fracture surface morphology, by keeping the realization of the random distribution the same for all the plates. The focus is kept on the two extreme cases in terms of the number of nucleation sites, with $N_{\mathrm{p}}=10$ for Fig. 10 and $N_{\mathrm{p}}=100$ for Fig. 11, while the size range is $k \in[3,6,9,15,18]$. For the plates in Fig. 10 (Fig. 11), the spatial distribution of the nucleation sites is also the same as the plate shown in Fig. 4 (Fig. 6). For $k=3$, the fracture surface morphology is of a cup-cup type both for $N_{\mathrm{p}}=10$ (Fig. 4) and $N_{\mathrm{p}}=100$ (Fig. 11a). For larger $k$ values, the fracture surface morphology shifts to a cup-cone form for $N_{\mathrm{p}}=10$, and the material separation takes place through the same set of interacting nucleation sites (see Fig. 10). For $N_{p}=100$, on the other hand, the fracture surface morphology shifts to a slant-like form for small void nucleation sites and remains slanted thereafter (see Fig. 11). As would be expected, the diffuse thinning region fades out and the fracture strain decreases with increasing $k$ for both $N_{\mathrm{p}}=10$ and $N_{\mathrm{p}}=100$.

\section{Discussion and Conclusions}

The FE framework adopted in this study allows the effects of the size, number, and spatial distribution of second phase particles to enter the analysis of ductile plate tearing by embedding discrete void nucleation sites in the FPZ. The results show that two local shear bands emanate from each void nucleation site as soon as the void volume fraction in the nucleation site reaches approximately $0.1 \%$. The two local shear bands are perpendicular to each other, one aligned at $45^{\circ}$ and the other at $-45^{\circ}$ with respect 
to the loading direction, and the plastic strain in the local shear bands is only slightly larger than that in the surrounding matrix at this early stage of deformation; just enough to make the shear bands apparent. Upon further monotonic loading, plasticity increases everywhere in the plate, but intensifies the most in the local shear bands spreading out from the void nucleation sites. At some point during the loading, an interaction between the individual void nucleation sites sets in. The mechanism through which this interaction takes place depends greatly on the size, number, and spatial distribution of the nucleation sites, as well as on the porosity in the matrix surrounding the sites. ${ }^{2}$ The interaction mechanisms between void nucleation sites can be classified into three distinct types: (I) The existing local $\pm 45^{\circ}$ shear bands spreading out from several nucleation sites interact with one another in such a way as to form a global localized deformation band extending over all these nucleation sites; see e.g. Fig. 6. (II) New shear bands form to connect neighboring void nucleation sites; see e.g. Figs. 12a and 12b. This mechanism is also known as the "void sheeting mechanism", where two existing large voids are linked by a shear localization band in which new voids can originate from secondary particles (see e.g. Cox and Low, 1974). (III) Neighboring void nucleation sites merge with one another through local necking of the ligament situated between them; see e.g. Figs. 12c and 12d.

Mechanism (I) is active only if a macroscopic localization band can evolve due to the interaction between the nucleation sites in the FPZ, and this is most likely to be the case if the number or size (or both) of the void nucleation sites is large enough. If active, mechanism (I) sets in early in the deformation history, with no or negligible diffuse thinning of the plate (see e.g. Fig. 7). In contrast, if the FPZ contains only a few small void nucleation sites, a macroscopic localization band cannot be achieved yielding a transient in the fracture surface morphology. In such plates, the interaction between nucleation sites, if it ever takes place, is postponed until after considerable diffuse thinning of the FPZ has developed. The plate shown in Fig. 4

\footnotetext{
${ }^{2}$ Note again that the background porosity in the matrix represents the presence of a secondary population of particles with much smaller sizes compared to the primary population of particles represented by the nucleation sites, see Section 2. In this study, the focus is on the primary population, and therefore the volume fraction of the secondary particles is assumed to be very small.
} 
is a good example of the case where no interaction takes place between nucleation sites, and the crack spreads by the growth of a single nucleation site captured in the diffuse necking zone. A fracture process involving only a single void has recently been reported in an experimental study by Noell et al. (2018), for tensile testing of a sheet specimen fabricated from a high-purity (99.999\%) polycrystalline aluminum. Depending on the spatial distribution, however, more than one void nucleation site might be involved in crack propagation, joining together through mechanism (II) (see Figs. 5b and 5c). For intermediate levels of both the number and size of the void nucleation sites, even if there exists a $45^{\circ}$ path extending over some nucleation sites, the path is usually not long enough to extend the entire plate thickness. The tearing crack can thereby propagate either through two intersecting global $45^{\circ}$ shear bands, leading to a cup-cone morphology (see Fig. 8b), or by a combination of all three micromechanisms (see Fig. 8d). Clear examples of mechanism III are only observed between void nucleation sites located in close proximity, which reveals that the interaction between void nucleation sites in relation to plate tearing predominantly occurs via shear bands.

An issue that is closely related to the crack tearing mechanisms is the question of whether macroscopic localization occurs prior to microscopic localization, or whether the two occur simultaneously. In macroscopic localization, in either a normal band or a shear band, the softening giving rise to localized deformation is linked to the nucleation and growth of the voids. In microscopic localization, however, the softening is due to the coalescence of the voids where plastic strain is confined in the ligaments connecting neighboring voids. Tekog̃lu et al. (2015) addressed this question by modeling an infinite planar band containing a doubly periodic array of initially spherical voids, embedded between two semi-infinite blocks of uniform, void-free material, in a three-dimensional FE setting. In Tekog̃lu et al. (2015) it was concluded that; macroscopic localization precedes microscopic localization (or void coalescence) at stress triaxilities larger than 1, as microscopic localization requires additional plastic straining in the deformation band. At lower stress triaxiality, however, the two modes of plastic flow localization occur simultaneously. In the present FE simulations of steady-state ductile tearing, the stress triaxiality is approximately 0.6 in the FPZ and it is clearly observed that macro- 
scopic localization will precede microscopic localization. Only if failure takes place by the first mechanism, as discussed above, will the localized deformation in a macroscopic shear band occur simultaneously with the onset of void coalescence. ${ }^{3}$ However, this is the case only when the number and/or size of the nucleation sites are large enough. For intermediate configurations of the damage related microstructure, macroscopic localization precedes microscopic localization, which usually takes place through void sheeting (mechanism (II)), and rarely through coalescence of neighboring nucleation sites (mechanism III)). With decreasing number and/or size of nucleation sites, the additional plastic straining in the localization band required to trigger microscopic localization increases, and the separation between the two modes of localization becomes increasingly clear. As discussed in Tekoğlu et al. (2015), in their FE model: i) the voids are highly aligned, which possibly advances void coalescence, and ii) the outer blocks are void free, which postpones macroscopic localization; the combination of both effects leads to simultaneous occurrence of the two modes at stress triaxialities below 1. If these two restrictions are removed, as is the case for the present FE framework, macroscopic localization precedes microscopic localization even at triaxiality values as low as 0.6 , except if the strain hardening capacity of the material is very low (in the present study, due to a large volume fraction of void nucleation sites).

In essence, what determines the fracture surface morphology is the strain hardening capacity of the plate material. This study emphasizes the fact that the strain hardening capacity of a material depends not only on the strain hardening exponent of the matrix, but also on the volume fraction and spatial distribution of the second phase particles, as well as on the ease of void nucleation at particle sites. Increasing the volume fraction of void nucleating particles would lower the hardening capacity of a material, the effect being more pronounced for easily void nucleating particles. The material parameters in this study are chosen in such a way to cover a broad spectrum

\footnotetext{
${ }^{3}$ It is worth noting that void coalescence within the elements inside a nucleation site, which occurs prior to macroscopic localization, is not the microscopic localization discussed here. The formation of a hole at a void nucleation site through coalescing voids simply mimics void nucleation at a particle, which, in reality, occurs either via brittle fracture of the particle, or the separation of the interface between the particle and the surrounding matrix.
} 
of hardening capacities, by only changing the number, $N_{\mathrm{p}}$, size, $R_{\mathrm{p}}$, and spatial distribution of the void nucleation sites, while keeping the remaining parameters the same. At one end of the spectrum stand the plates with a low number of small nucleation sites, for which the hardening capacity is large and the crack predominantly propagates in a cup-cup morphology. At the other end are the plates with a large number of big nucleation sites, whose low hardening capacity forces the crack to slant. At these extremes of the spectrum, the crack profile depends mainly on the volume fraction of the nucleation sites; in the intermediate range, however, the spatial distribution is as important as the volume fraction.

The present study investigates only the effects of the size, number, and spatial distribution of second phase particles on the crack profile, while assuming all the remaining mechanical and geometric parameters to be the same for all the cases analyzed. Employing, for example, a different set of material parameters could lead to a different fracture surface morphology for specific $N_{\mathrm{p}}-R_{\mathrm{p}}$ combinations, especially for intermediate values. Although the general trends discussed above are expected to stand their ground, the mechanical properties of the matrix and the particles as well as the shape of the particles would affect when the transition form one crack morphology to another occurs. It will be, therefore, important to address this issue in the future. In fact, an attempt into this direction has been made in a recent work presented in Kaçar et al. (2017), which shows that the crack profile shifts from slanted to cup-cup with increasing matrix strain hardening capacity, in accordance with experiments.

Finally, it is worth noting that although the presented FE framework is suitable for the qualitative analyses performed in this study, a quantitative comparison with experiments would require a more advance ductile fracture model accounting for the effects of parameters such as void shape, anisotropic mechanical properties, kinematic hardening, etc.

\section{Acknowledgments}

The authors gratefully acknowledge the financial support by TÜBİTAK (Project No: 315M133). KLN is financially supported by the VILLUM FOUNDATION Young Investigator Programme in the project "A New phenomenon Yet to be resolved in ductile 
PLATE tearing", grant VKR023451.

\section{References}

ABAQUS, 2016. Abaqus documentation collection, Version 2016, Providence, RI: Dassault Systmes.

Andersen, R., Felter, C., Nielsen, K., 2018a. Micro-mechanics based cohesive zone modeling of full scale ductile plate tearing: from initiation to steady state. Submitted for publication.

Andersen, R., Woelke, P., Nielsen, K., 2018b. Cohesive traction-separation relations for plate tearing under mixed mode loading. Eng. Frac. Mech. 71, 199-209.

Asserin-Lebert, A., Besson, J., Gourgues, A. F., 2005. Fracture of 6056 aluminum sheet materials: effect of specimen thickness and hardening behavior on strain localization and toughness. Materials Science and Engineering: A 395, 186 - 194.

Benzerga, A. A., Leblond, J.-B., 2010. Ductile fracture by void growth to coalescence. Adv. Appl. Mech. 44, 169-305.

Besson, J., Steglich, D., Brocks, W., 2003. Modeling of plane strain ductile rupture. Int. J. Plasticity 19, 1517-1541.

Broek, D., 1986. Elementary Engineering Fracture Mechanics. Kluwer Academic Publishers, Netherlands.

Bron, F., Besson, J., Pineau, A., 2004. Ductile rupture in thin sheets of two grades of 2024 aluminum alloy. Materials Science and Engineering: A 380 (1), 356 - 364.

Cox, T. B., Low, J. R., 1974. An investigation of the plastic fracture of AISI 4340 and 18 Nickel-200 grade maraging steels. Metall. Trans. 5 (6), 1457-1470.

Dighe, M. D., Gokhale, A. M., Horstemeyer, M. F., 2002. Second phase cracking and debonding observations in the fatigue damage evolution of a cast $\mathrm{Al}-\mathrm{Si}-\mathrm{Mg}$ alloy. Metall. Mater. Trans 33A, $1-8$. 
Felter, C. L., Nielsen, K. L., 2017. Assisted crack tip flipping under mode I thin sheet tearing. Eur. J. Mech. A/Solids 64, 58-68.

Gurland, J., 1972. Observations on the fracture of cementite particles in a spheroidized $1.05 \% \mathrm{C}$ steel deformed at room temperature. Acta Metallurgica 20, $735-741$.

Gurson, A., 1977. Continuum theory of ductile rupture by void nucleation and growth - part I: yield criteria and flow rules for porous ductile media. ASME J. Eng. Mater. Technol. 99, 2-15.

Irwin, G. R., Kies, J. A., Smith, H. L., 1958. Fracture strengths relative to onset and arrest of crack propagation. In: Proceedings of ASTM International. Vol. 58. pp. 640-60.

Kaçar, F., Tekog̃lu, C., Nielsen, K. L., 2017. Fracture surface morphology under ductile tearing of metal plates. In: Høgsberg, J., Pedersen, N. L. (Eds.), Proceedings of the 30th Nordic Seminar on Computational Mechanics, Kgs. Lyngby, Denmark. pp. 193 $-196$.

Knott, J. F., 1973. Fundamentals of Fracture Mechanics. Butterworths, London.

Lewandowski, J. J., Liu, C., Hunt, W. H., 1989. Effects of matrix microstructure and particle distribution on fracture of an aluminum metal matrix composite. Materials Science and Engineering: A 107, $241-255$.

Li, W., Siegmund, T., 2002. An analysis of crack growth in thin-sheet metal via a cohesive zone model. Engineering Fracture Mechanics 69, 2073 - 2093.

Marino, B., Mudry, F., Pineau, A., 1985. Experimental study of cavity growth in ductile rupture. Engineering Fracture Mechanics 22, 989 - 996.

Mathur, K., Needleman, A., Tvergaard, V., 1996. Three dimensional analysis of dynamic ductile crack growth in a thin plate. J. Mech. Phys. Solids 44 (3), 439-464.

Morgeneyer, T. F., Taillandier-Thomas, T., Buljac, A., Helfen, L., Hild, F., 2016. On strain and damage interactions during tearing: 3D in situ measurements and sim- 
ulations for a ductile alloy (AA2139-T3). Journal of the Mechanics and Physics of Solids $96,550-571$.

Morgeneyer, T. F., Taillandier-Thomas, T., Helfen, L., Baumbach, T., Sinclair, I., Roux, S., Hild, F., 2014. In situ 3-D observation of early strain localization during failure of thin $\mathrm{Al}$ alloy (2198) sheet. Acta Materialia 69, 78 - 91 .

Nielsen, K., Hutchinson, J., 2012. Cohesive traction-separation laws for tearing of ductile metal plates. Int. J. Imp. Eng. 48, 15-23.

Noell, P. J., Carroll, J. D., Boyce, B. L., 2018. The mechanisms of ductile rupture. Acta Mater. 161, 83 - 98.

Osovski, S., Srivastava, A., Ponson, L., Bouchaud, E., Tvergaard, V., Ravi-Chandar, K., Needleman, A., 2015. The effect of loading rate on ductile fracture toughness and fracture surface roughness. J. Mech. Phys. Solids 76, $20-46$.

Pardoen, T., Hachez, F., Marchioni, B., Blyth, P. H., Atkins, A. G., 2004. Mode I fracture of sheet metal. J. Mech. Phys. Solids 52, $423-452$.

Srivastava, A., Osovski, S., Needleman, A., 2017. Engineering the crack path by controlling the microstructure. J. Mech. Phys. Solids 100, $1-20$.

Srivastava, A., Ponson, L., Osovski, S., Bouchaud, E., Tvergaard, V., Needleman, A., 2014. Effect of inclusion density on ductile fracture toughness and roughness. J. Mech. Phys. Solids 63, $62-79$.

Tekog̃lu, C., Hutchinson, J., Pardoen, T., 2015. On localization and void coalescence as a precursor to ductile fracture. Phil. Trans. R. Soc. A373.

Tvergaard, V., 1981. Influence of voids on shear band instabilities under plane strain condition. Int. J. Fract. Mech. 17, 389-407.

Tvergaard, V., 1990. Material failure by void growth to coalescence. Adv. Appl. Mech 27, 83-151. 


\section{Appendix}

Rather than considering the equivalent plastic strain, the evolution of damage is used as an indicator of localization throughout the present work. This choice is motivated by the fact that the localized deformation regions are clearly visible in both contour plots: compare Figs. A-1a and A-1b, or Figs. A-1c and A-1d. All four figures correspond to the onset of macroscopic localization, Figs. A-1a and A-1b for a plate with $N_{\mathrm{p}}=10, k=3$, and Figs. A-1a and A-1b for a plate with $N_{\mathrm{p}}=100, k=18$.

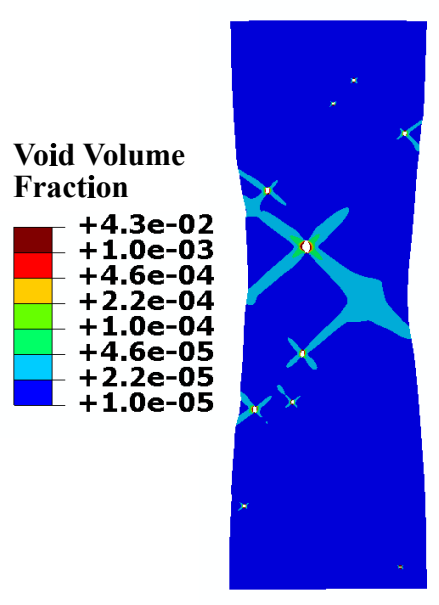

(a)

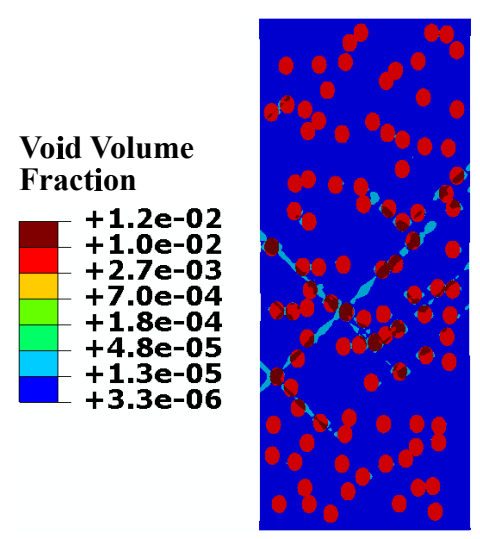

(c)

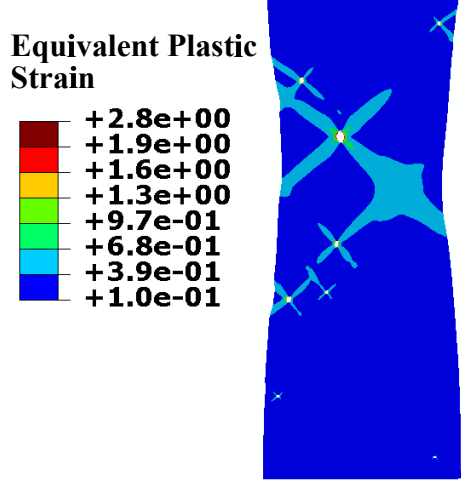

(b)
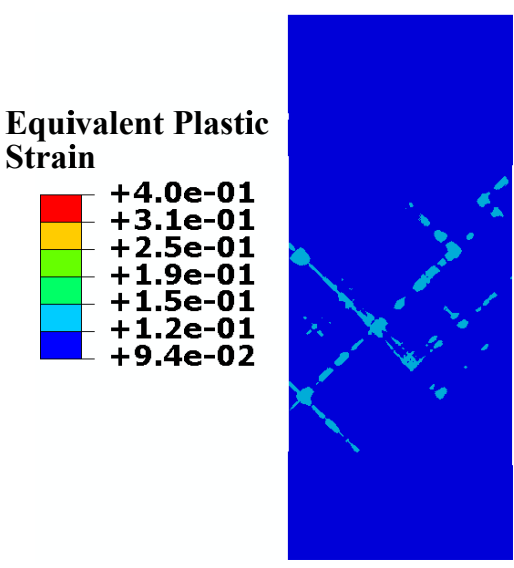

(d)

Figure A-1: Developed $(\mathrm{a}, \mathrm{c})$ void volume fraction and $(\mathrm{b}, \mathrm{d})$ equivalent plastic strain in the fracture process zone at the onset of macroscopic localization. The plate in (a,b), with $N_{\mathrm{p}}=10, k=3$, is also shown in Fig. 4, and the plate in (c,d), with $N_{\mathrm{p}}=100, k=18$, is also shown in Fig. 6 . 


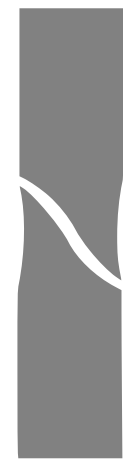

(a)

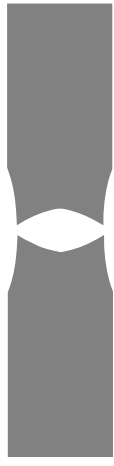

(b)

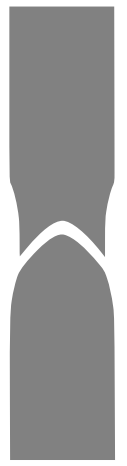

(c)

Figure 1: Schematic showing cross sections of cracked plate cross sections with the crack growing toward the reader: (a) the slanted crack growth, (b) the cup-cup crack growth (or bath tub), and (c) the cup-cone crack growth (or double slant).

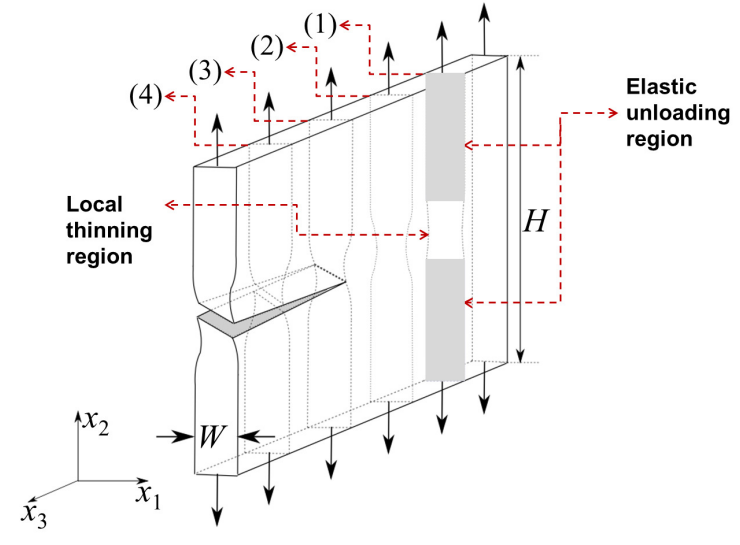

(a)

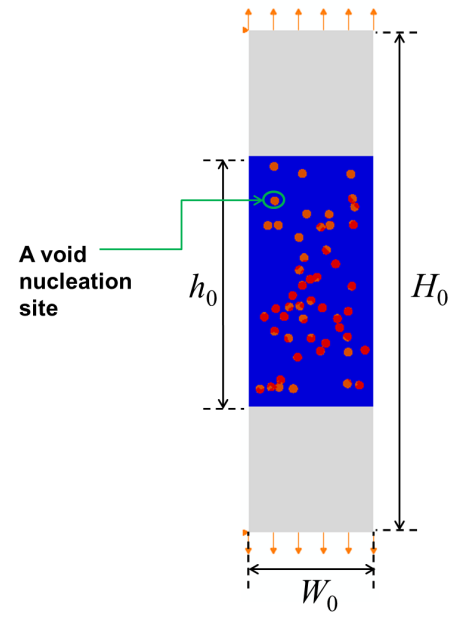

(b)

Figure 2: Schematic illustrating (a) a ductile plate under mode I loading, and (b) a 2D plane strain FE model for the plate in the undeformed configuration. Void nucleation sites in the fracture process zone (with a height of $h_{0}$ ) represent the second phase particles in a real material. 


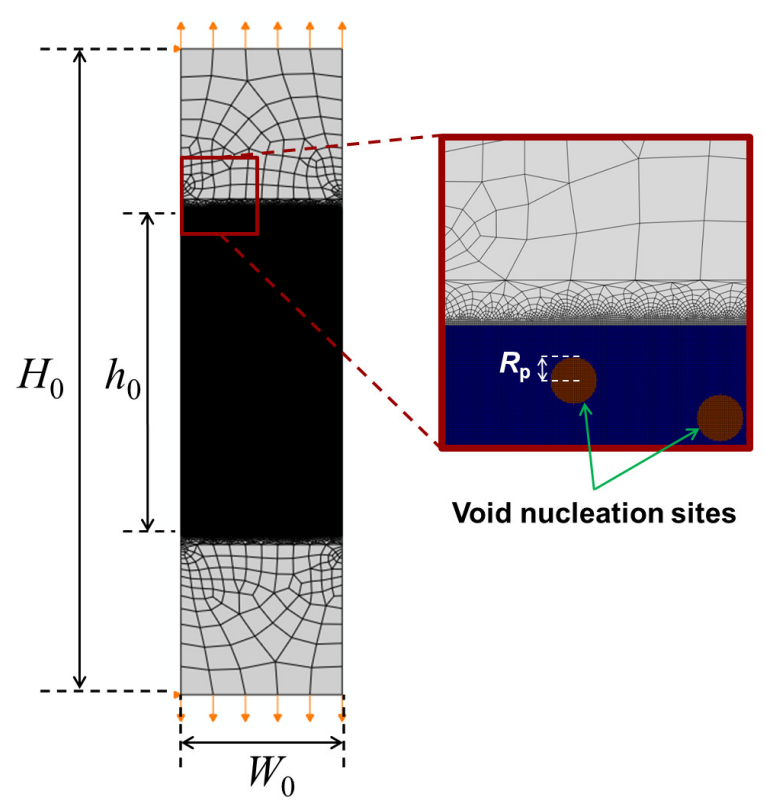

Figure 3: Schematic showing the boundary conditions and a typical mesh for the 2D plane strain FE model for a ductile plate under mode I loading. Void nucleation sites have an initial circular shape with a radius of $R_{\mathrm{p}}$, as shown in the magnified view at the right. Mode I loading is imposed on the plate by pulling the top and bottom boundaries in the vertical direction, while letting them remain traction free in the horizontal direction. Moreover, the horizontal displacements of the two nodes located at the top-left and bottom-left corners are fixed, so that the top and bottom parts of the plate do not move in the horizontal direction with respect to one another once the crack starts to propagate. 


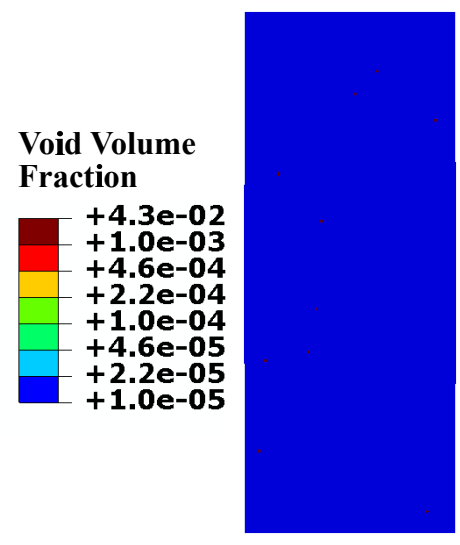

(a)

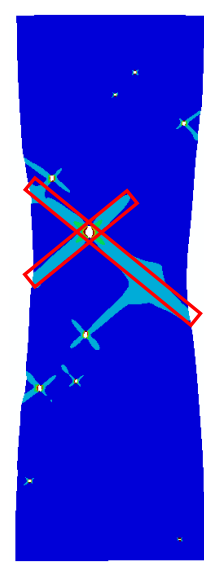

(b)

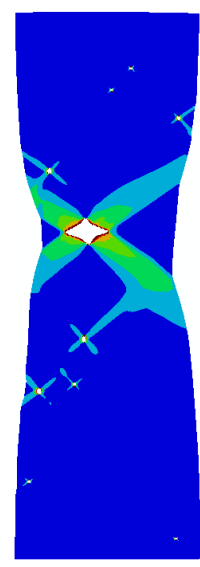

(c)

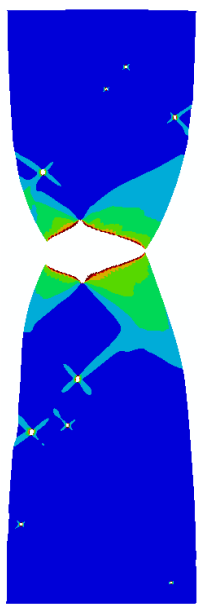

(d)

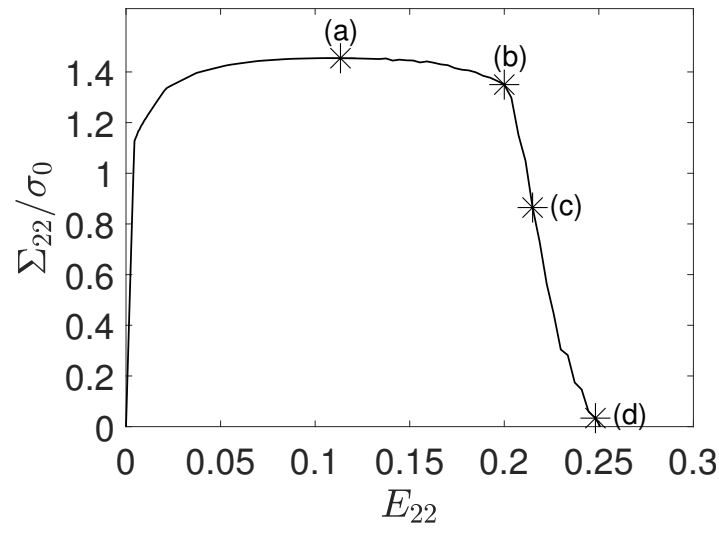

(e)

Figure 4: Developed void volume fraction in the fracture process zone for a plate with $N_{\mathrm{p}}=10, k=3$, at (a) the onset of diffuse thinning, (b) the onset of macroscopic localization, (c) an intermediate deformation stage between the onset of macroscopic localization and final failure, and (d) the final failure. (e) Variation of the normalized engineering stress $\Sigma_{22} / \sigma_{0}$ with the engineering strain $E_{22}$ for the plate. 


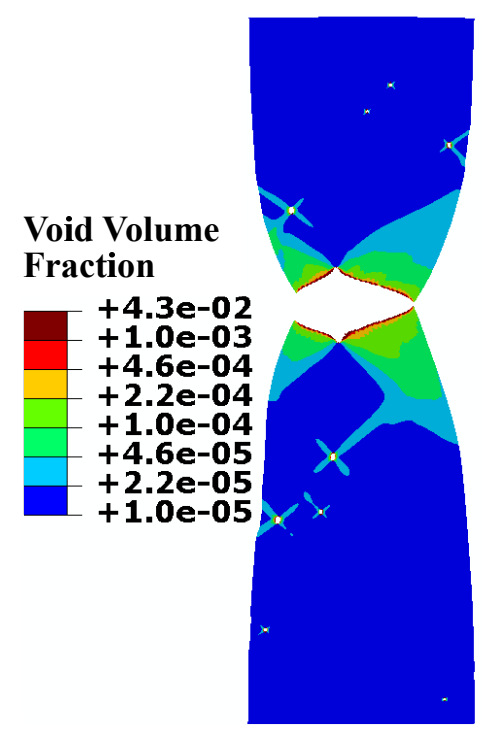

(a) Plate P1

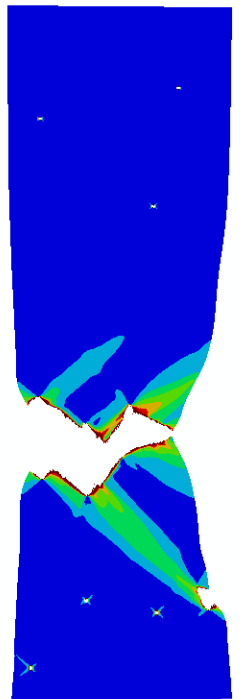

(b) Plate P2

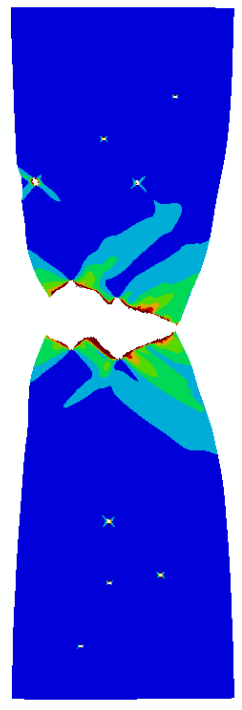

(c) Plate P3

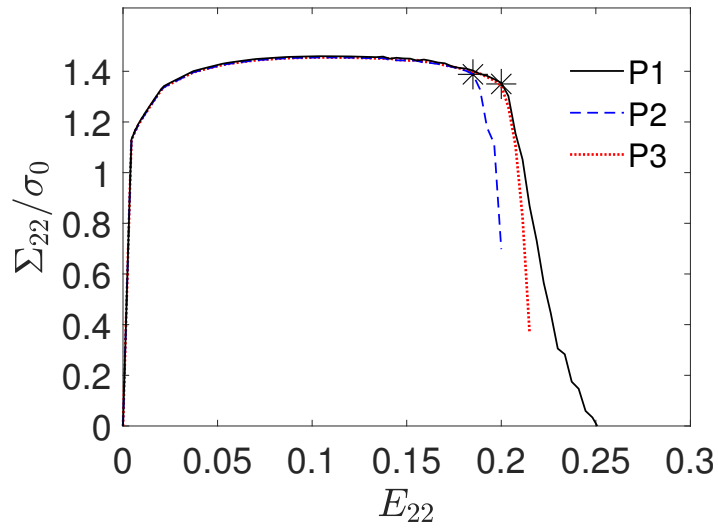

(d)

Figure 5: Developed void volume fraction in the fracture process zone for three plates with $N_{\mathrm{p}}=$ $10, k=3$ at the final failure: (a) plate $\mathrm{P} 1$, (b) plate $\mathrm{P} 2$, and (c) plate P3. Plate P1 is also shown in Fig. 4. All the material parameters for these three plates are the same, the only difference being the realization of randomly distribution void nucleation sites. (d) Variation of the normalized engineering stress $\Sigma_{22} / \sigma_{0}$ with the engineering strain $E_{22}$ for the three plates. 


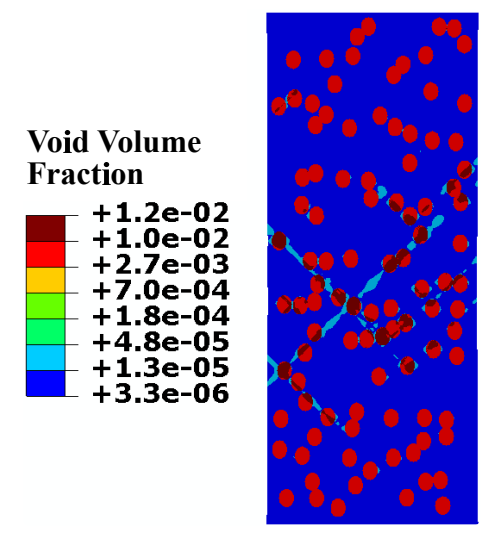

(a)

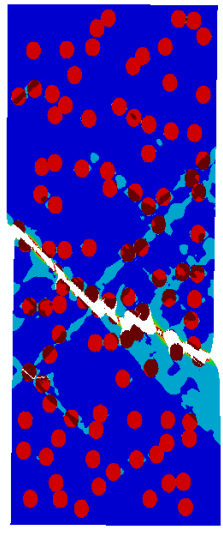

(b)

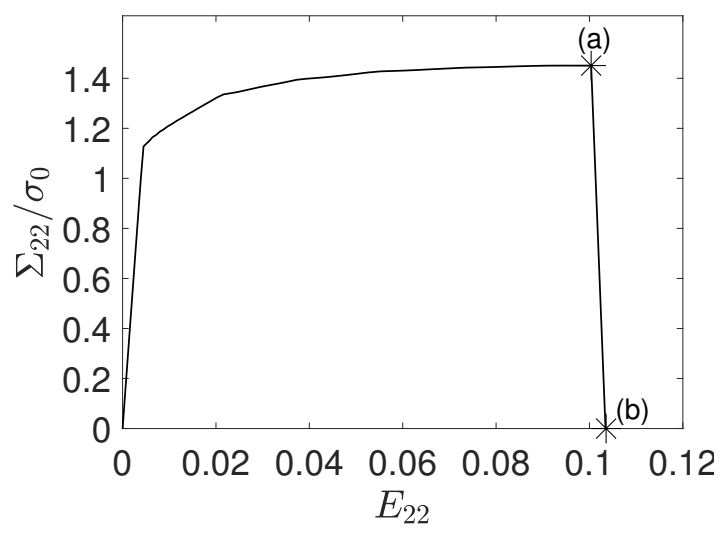

(c)

Figure 6: Developed void volume fraction in the fracture process zone for a plate with $N_{\mathrm{p}}=100, k=18$ at the (a) onset of macroscopic localization, and (b) final failure. (c) Variation of the normalized engineering stress $\Sigma_{22} / \sigma_{0}$ with the engineering strain $E_{22}$ for the plate. 


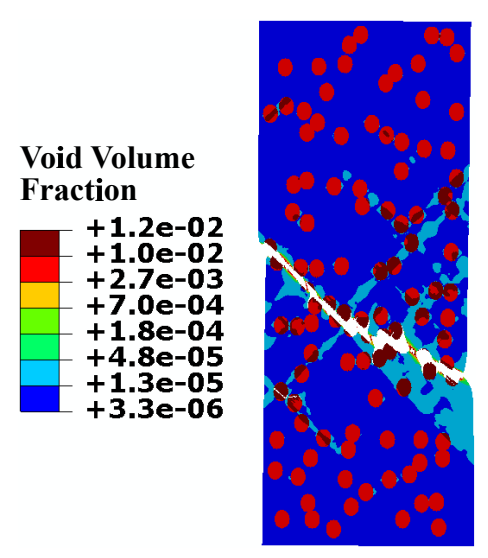

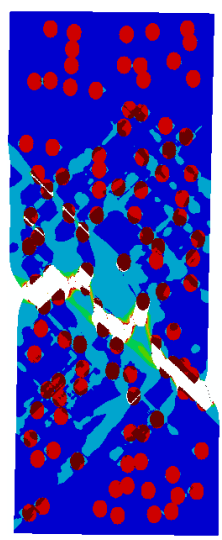

(b) Plate P5

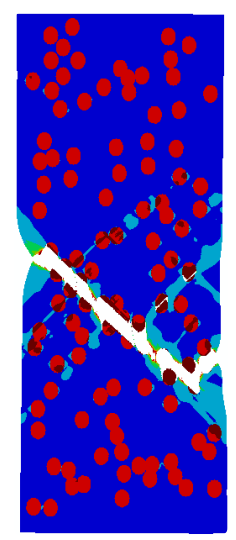

(c) Plate P6

(a) Plate P4

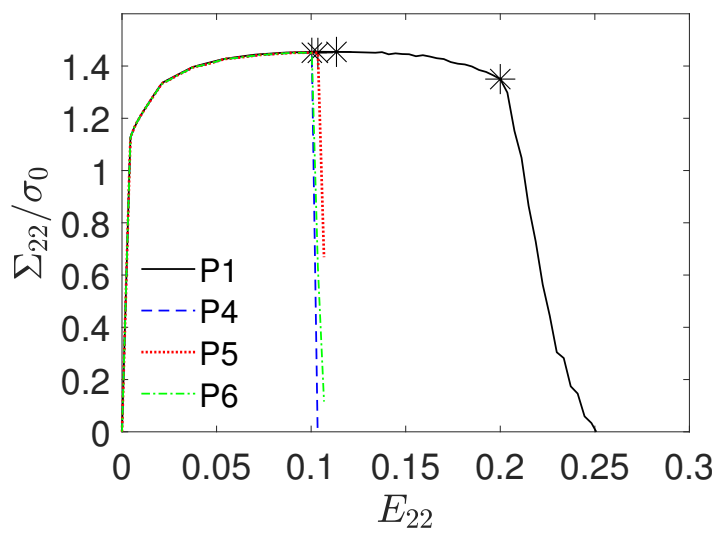

(d)

Figure 7: Developed void volume fraction in the fracture process zone for three plates with $N_{\mathrm{p}}=$ $100, k=18$ at the final failure: (a) plate $\mathrm{P} 4$, (b) plate $\mathrm{P} 5$, and (c) plate P6. Plate P4 is also shown in Fig. 6. All the material parameters for these three plates are the same, the only difference being the spatial distribution of the void nucleation sites. (d) Variation of the normalized engineering stress $\Sigma_{22} / \sigma_{0}$ with the engineering strain $E_{22}$ for the plates P4, P5, P6 and the plate P1 (with $N_{\mathrm{p}}=10, k=3$ ) shown in Fig. 4. 


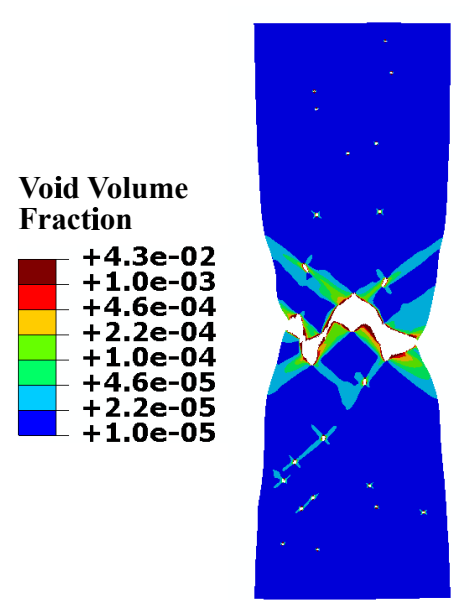

(a)

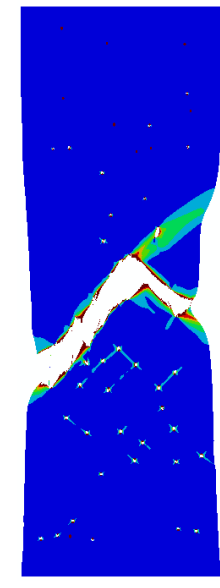

(b)

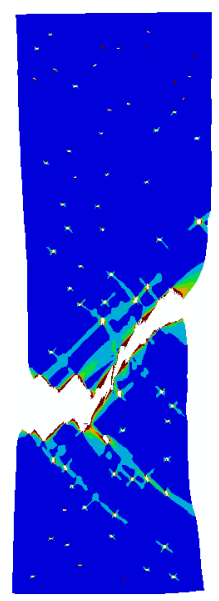

(c)

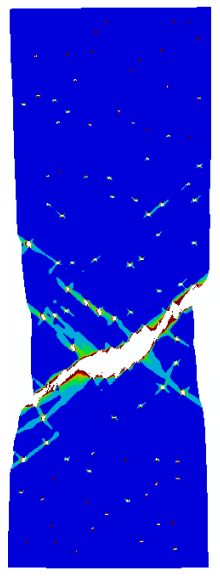

$(\mathrm{d})$

Figure 8: Developed void volume fraction in the fracture process zone for four different plates. The size of the void nucleation sites is the same for all the four plates $(k=3)$, while their number is different: (a) $N_{\mathrm{p}}=25$, (b) $N_{\mathrm{p}}=50$, (c) $N_{\mathrm{p}}=75$, and (d) $N_{\mathrm{p}}=100$.

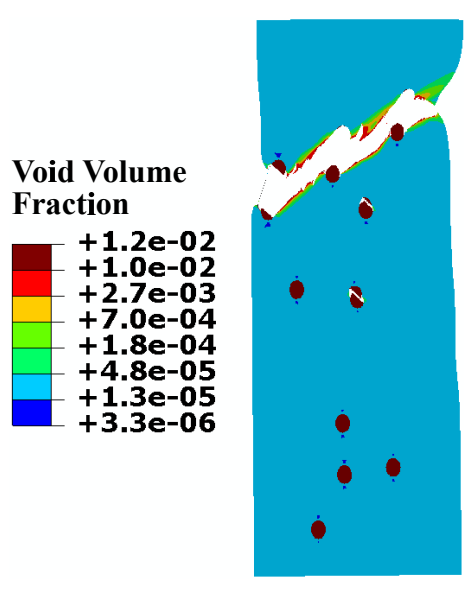

(a)

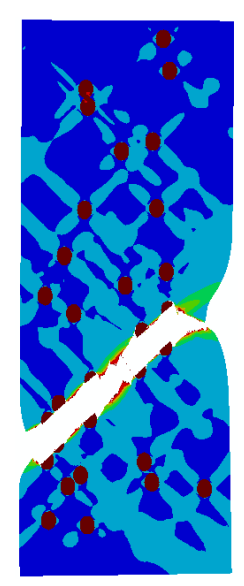

(b)

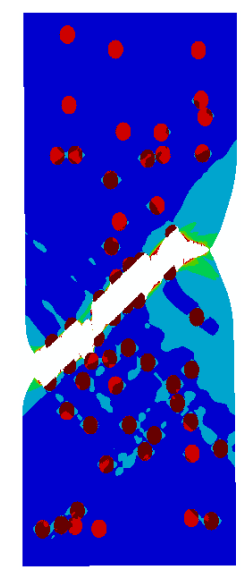

(c)

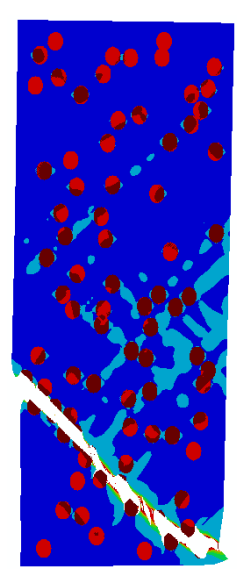

(d)

Figure 9: Developed void volume fraction in the fracture process zone for four different plates. The size of the void nucleation sites is the same for all the four plates $(k=18)$, while their number is different: (a) $N_{\mathrm{p}}=10$, (b) $N_{\mathrm{p}}=25$, (c) $N_{\mathrm{p}}=50$, and (d) $N_{\mathrm{p}}=75$. 


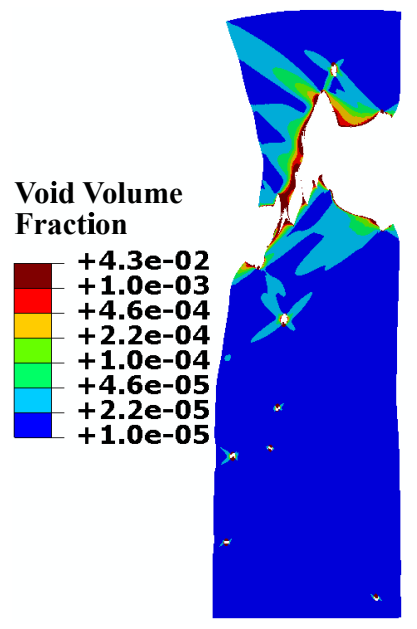

(a)

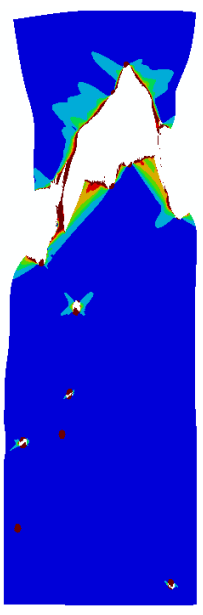

(b)

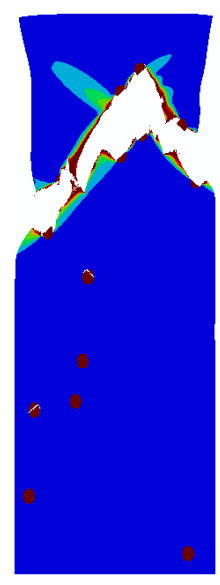

(c)

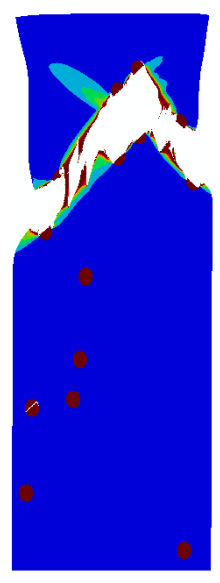

(d)

Figure 10: Developed void volume fraction in the fracture process zone for four different plates. The spatial distribution and the number of the void nucleation sites are the same for all the four plates $\left(N_{\mathrm{p}}=10\right.$ ), while their size is different: (a) $k=6$, (b) $k=9$, (c) $k=15$, and (d) $k=18$.

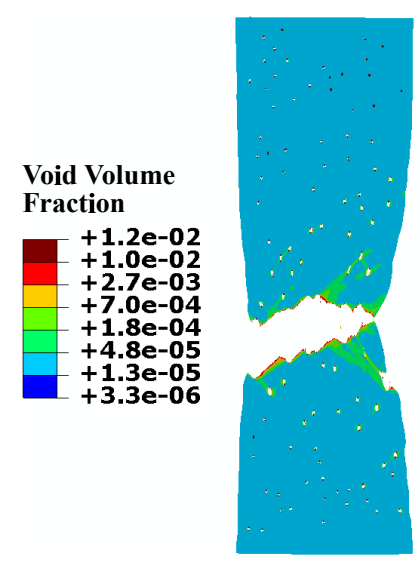

(a)

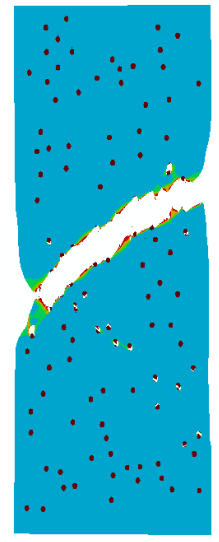

(b)

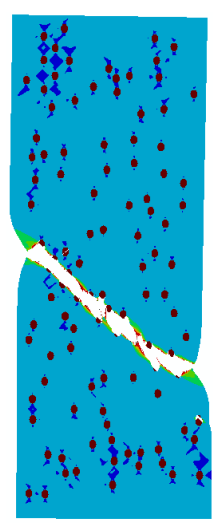

(c)

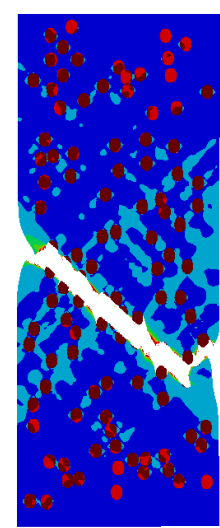

(d)

Figure 11: Developed void volume fraction in the fracture process zone for four different plates. The spatial distribution and the number of the void nucleation sites are the same for all the four plates $\left(N_{\mathrm{p}}=100\right.$ ), while their size is different: (a) $k=3$, (b) $k=6$, (c) $k=9$, and (d) $k=15$. 


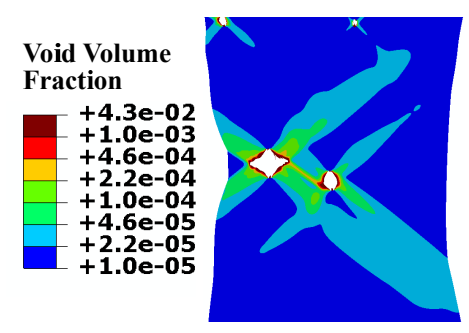

(a)

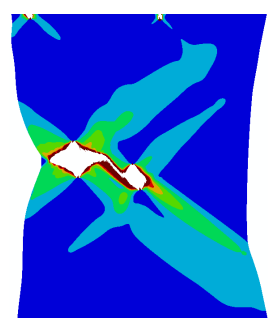

(b)

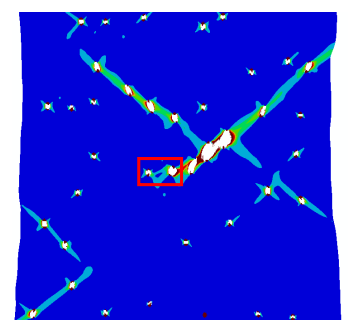

(c)

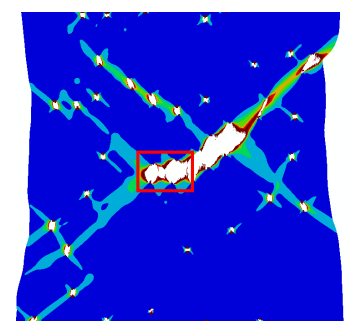

(d)

Figure 12: (a) Onset of void sheeting and (b) separation of the matrix trough void sheeting between two void nucleation sites. (c) Onset of coalescence and (d) internal necking of the ligament between two void nucleation sites. The plate in $(a, b)$ is also shown in Fig. $5 c$, and the one in $(c, d)$ is also shown in Fig. 8d. 